Trauma Berufskrankh 2004 - 6 [Suppl 3] : S293-S295 DOI 10.1007/s10039-004-0909-2

Online publiziert: 17. Juni 2004

(c) Springer-Verlag 2004
L. Oezokyay · G. Muhr · F. Kutscha-Lissberg

Chirurgische Klinik und Poliklinik, BG-Kliniken Bergmannsheil, Universitätsklinik Bochum

\title{
Anwendung winkelstabiler Implantate am distalen Radius
}

ie distale Radiusfraktur stellt die häufigste Fraktur des Menschen in allen Alterspopulationen dar. Bei jungen Patienten tritt sie als Kombinationsverletzung mit Polytrauma [5] bzw. als Folge von Hochrasanztraumen, wie z. B. Inline-Skating [4], auf. Beim älteren Patienten führen schon Bagatelltraumen zur distalen Radiusfraktur, begünstig durch eine osteoporotische Knochenstruktur. Mit einem Anteil von $17 \%$ aller Frakturen und einer Inzidenz von 2:10oo Einwohnern/Jahr ist sie die in der unfallchirurgischen Praxis am häufigsten zu behandelnde Verletzung [7].

Aufgrund des Problems der Retention und der häufigen sekundären Dislokation bzw. Redislokation unter konservativer Behandlung stellt diese häufige Fraktur mit in 20-30\% unbefriedigenden Ergebnissen nach wie vor eine Herausforderung der modernen Unfallchirurgie dar [9].

\section{Behandlung}

Die Therapie der distalen Radiusfraktur dient der Wiederherstellung des radioulnaren Gelenks, der Längenkongruenz sowie der Rekonstruktion der radiokarpalen Gelenkfläche mit entsprechender Ausrichtung des Gelenks [10]. Einfache extraartikuläre Frakturformen können konservativ im Gipsverband therapiert werden, ggf. ist vorher eine geschlossene Reposition im Aushang erforderlich.

Extraartikuläre Frakturen mit Trümmerzone oder komplexe intraartikuläre Frakturen bedürfen einer operativen Ver- sorgung, die dem jeweiligen Frakturtyp angepasst werden muss. Die operativen Therapiemöglichkeiten umfassen

$$
\begin{aligned}
& \text { - perkutane Kirschner-Draht-Osteo- } \\
& \text { synthese } \\
& \text { - Plattenosteosynthese } \\
& \text { - Fixateur-externe-Anlage } \\
& \text { - Kombinationen obiger Verfahren }
\end{aligned}
$$

Bei der Versorgung mittels Platte wurde in der Vergangenheit die Platte bei dorsaler Dislokation von streckseitig angebracht. Aufgrund der häufig zu beobachtenden Komplikationen wie Rupturen der Sehne des M. extensor pollicis longus und Verklebungen der streckseitigen Sehnenfächer hat sich ein Wandel zur beugeseitiger Plattenanlage vollstreckt $[3,8]$. Zudem sind in den letzten Jahren winkelstabile Implantate auf den Markt gekommen, die eine stabile Verankerung der Schrauben schon in der Platte durch ein Plattenlochgewinde ermöglichen. Dies erlaubt eine Versorgung der dorsal dislozierten Frakturen über einen volaren $\mathrm{Zu}$ gang ohne das Risiko einer sekundären Dislokation. Der beugeseitige Zugang wird in der Literatur als komplikationsärmer angegeben $[1,6]$. Bei übungsstabiler Osteosynthese ist bei dieser Versorgungsart die frühfunktionelle Mobilisation erlaubt und wünschenswert.

\section{Material und Methode}

In den BG-Kliniken Bergmannsheil Bochum wurden zwischen 1.10.2000 und
31.12.2002 103 Patienten mit distaler Radiusfraktur mittels winkelstabiler volarer Plattenosteosynthese (Fa. Königsee, Aschau) versorgt. Wir konnten $101 \mathrm{~Pa}$ tienten nach 11-37 Monaten klinisch und radiologisch nachuntersuchen. Das betroffene Handgelenk wurde röntgenologisch untersucht, das Röntgenbild wurde mit den Unfall- und postoperativen Aufnahmen verglichen. Die Röntgenbilder wurden vermessen, mit Bestimmung der Gelenkwinkel a.-p. und seitlich, Ulnavorschub, radialem Shift und radialer Höhe. Die Patienten wurden klinisch untersucht und zur subjektiven Zufriedenheit befragt. Anschließend führten wir eine Ballonmanometeruntersuchung zur Kraftentwicklung mit Vergleich zur Gegenseite durch.

Von den 101 Patienten waren 26 Männer und 75 Frauen, das Alter zum Unfallzeitpunkt lag bei durchschnittlich 59,7 Jahren (20,3-92,3 Jahren). Es war 41-mal die rechte und 60-mal die linke Seite betroffen. Nach der AO-Klassifikation lagen folgende Frakturen vor:

$$
\begin{aligned}
& -2 \mathrm{~A} 2 \text {-Frakturen } \\
& -39 \mathrm{~A} 3 \text {-Frakturen } \\
& -2 \mathrm{~B} 3 \text {-Frakturen } \\
& -23 \mathrm{C} 1 \text {-Frakturen } \\
& -8 \mathrm{C} 2 \text {-Frakturen } \\
& -27 \mathrm{C} 3 \text {-Frakturen }
\end{aligned}
$$

Unfallursachen waren in 73 Fällen ein Sturz in der Ebene, 13-mal ein Sportunfall, 10 Patienten hatten einen Sturz aus weniger als 5 m Höhe, 2 Patienten stürzten 
Trauma Berufskrankh 2004 - 6 [Suppl 3] : S293-S295

DOI 10.1007/s10039-004-0909-2

C) Springer-Verlag 2004

\section{L.Oezokyay · G. Muhr · F. Kutscha-Lissberg}

\section{Anwendung winkelstabiler Implantate am distalen Radius}

\section{Zusammenfassung}

Die optimale Therapie der distalen Radiusfraktur sollte zur anatomischen Reposition und freien, schmerzlosen Handgelenkbeweglichkeit führen. Unbefriedigende radiologische und anatomische Ergebnisse treten jedoch nach Tscherne bei 20 $30 \%$ der Fälle auf.Im Rahmen einer retrospektiven Studie wurden 101 Patienten nach 11-37 Monaten untersucht (klinische und radiologische Kontrolle), die mittels volarer winkelstabiler Titanplatte (Fa. Königsee) zwischen Oktober 2000 und Dezember 2002 operativ versorgt worden waren (A0-Klassifikation: 41 A-, 2 B-, 58 C-Frakturen). Als Komplikationen zeigten sich 2 EPL-Rupturen, 2-mal intraartikuläre Schraubenlage, 1 persistierender Ulnavorschub, der operativ mittels Kapandji-Sauvé therapiert wurde, sowie 3 post- operative CTS.76 Patienten waren mit der operativen Therapie sehr zufrieden und zeigten keine Einschränkung der Alltagsbelastung. Bei der Kraftmessung wurde bei 46 Patienten ein objektiver Kraftverlust zwischen 10 und 60\% zur gesunden Gegenseite festgestellt. Die volare winkelstabile Plattenosteosynthese bei distalen extra- und intraartikulären Radiusfrakturen führt zu einer sicheren operativen Versorgung bei Beachtung der implantatspezifischen Eigenschaften.

\section{Schlüsselwörter}

Distale Radiusfraktur · Winkelstabilität · Volarer Zugang · Gelenkwinkel

\section{Fixed-angle titanium plates in fractures of the distal radius}

\begin{abstract}
Optimal treatment of a fracture of the distal radius should lead to anatomical reduction and unrestricted, pain-free mobility of the wrist. According to Tscherne, however, radiological and anatomical results are unsatisfactory in about $20-30 \%$ of cases. In a retrospective study we examined 101 patients 11-37 months after insertion of fixed-angle titanium plates (Königsee, Aschau, Germany) by a volar approach. The initial operations had been performed from October 2000 to December 2002. The fractures treated were classed as A in 41 cases, B in 2 and C in 58 (A0 classification). Postoperative complications seen were EPL ruptures (2 cases), intra-articular position of the screw (2 cases), persisting ulnar
\end{abstract}

protrusion corrected by a Kapandji-Sauvé procedure (1) and postoperative CTS (3). In 76 cases the patients were very satisfied with the outcome of surgery and were not restricted in their everyday lives. Strength measurements revealed an objective loss of 10-60\% of the compared with the healthy contralateral arm in 46 patients. Fixed-angle internal fixation of distal extra- and intra-articular radial fractures by a volar approach is an adequate treatment when enough attention is paid to the implant-specific properties.

\section{Keywords}

Distal radius fracture · Fixed angle $\cdot$ Volar approach · Joint angle aus mehr als $5 \mathrm{~m}$ und 3 Patienten verunfallten im Rahmen eines Verkehrsunfalls.

Innerhalb der ersten $24 \mathrm{~h}$ nach dem Trauma wurden 66 Patienten versorgt, der längste Abstand zwischen Unfall und operativer Versorgung betrug 19 Tage. Bei 8 Patienten wurde zusätzlich die Ulna mit versorgt, 2 Patienten erhielten zusätzlich eine Kirschner-Draht-Stabilisierung in den distalen Radius. Bei 1 Patienten erfolgte am 1. postoperativen Tag bei frühzeitiger Frakturdislokation die additive dorsale Plattenosteosynthese.

In der Regel wurde postoperativ 4 Wochen im Gips ruhig gestellt, bei jungen $\mathrm{Pa}$ tienten wurde eine Schienenruhigstellung bis zur Wundheilung vorgenommen.

\section{Ergebnisse}

Bei der Auswertung der postoperativen Röntgenbilder zeigte sich bei 29 Patienten, dass nur 2 der 3 distalen winkelstabilen Schrauben regelrecht im Plattengewinde verankert waren. Als postoperative Komplikation trat in 2 Fällen eine Ruptur der Sehne des M. extensor pollicis longus nach 5 Wochen und 3 Monaten auf. Bei 2 Patienten fand sich eine intraartikuläre Schraubenlage, die mittels frühzeitiger Metallentfernung therapiert wurde. Bei 4 Patienten kam es zu einem Absinken des ulnaren Kantenfragments (Melone-Fragment), dies führte in 2 Fällen zu einer Stufenbildung der radialen Gelenkfläche von mehr als $2 \mathrm{~mm}$. Bei einem Patienten resultierte eine Persistenz des Ulnavorschubs, sodass nach 17 Monaten eine Operation nach Sauvé-Kapandji erforderlich wurde.

Bei dem Vergleich der Unfall- und Kontrollröntgenbildervermessung zeigte sich eine Korrektur des Radiusgelenkwinkels a.-p. von $6,3^{\circ}$, seitlich von $21,4^{\circ}$, der Ulnavorschub konnte um durchschnittlich $4 \mathrm{~mm}$ verkürzt, die radiale Höhe um $6 \mathrm{~mm}$ verbessert und die Radialverschiebung der distalen Speiche um 2 mm korrigiert werden. Bei keinem Patienten zeigte sich eine messbare Änderung der Gelenkwinkel vom postoperativen zum Kontrollröntgen, bei 27 Patienten ist bereits eine Metallentfernung erfolgt.

Bei der klinischen Untersuchung war bei 72 Patienten die Beweglichkeit zur gesunden Gegenseite eingeschränkt, ledig- 
lich 3 Patienten gaben an, diese Einschränkung behindere sie bei der Alltagstätigkeit.

76 Patienten sind mit dem erreichten Ergebnis zufrieden oder sehr zufrieden, 6 Patienten klagen über intermittierende Schmerzen, bei 3 Patienten zeigte sich klinisch ein Karpaltunnelsyndrom.

Bei Messung der Kraft mittels Ballonmanometer wiesen 55 Patienten eine seitengleiche Kraftentwicklung auf, $46 \mathrm{~Pa}$ tienten eine Kraftminderung Zwischen 10 und $60 \%$.

\section{Diskussion}

Die distale Radiusfraktur stellt die häufigste Fraktur beim Menschen dar, die mit bis zu 30\% unbefriedigende Therapieergebnisse bringt [9]. Die operative Therapie mittels volarer winkelstabiler Platte erweitert die Therapieoptionen. Der volare Zugang zum distalen Radius ist einfacher, die volare Plattenlage führt deutlich seltener zu Sehnenirritationen als die dorsale Lage. Mittels winkelstabiler volarer Platte kann die Reposition vor Plattenanlage oder über die Platte erfolgen, die Lage der ersten winkelstabilen Schraube bestimmt die weitere Schrauben- und Plattenlage. Es erscheint wichtig, die winkelstabilen Schrauben subchondral zu platzieren, um ein Nachsinken des distalen Fragments zu verhindern. Wir konnten in unserer Serie kein signifikantes Absinken feststellen.

Die winkelstabile volare Osteosynthese kann nicht alle distalen Radiusfrakturen beherrschen, v. a. ulnare Kantenfragmente werden häufig aufgrund ihrer Größe und Lage nicht suffizient mitgefasst, sodass hier weitere Implantate erforderlich werden.

In unserer Untersuchung kam es bei 2 Patienten zu Rupturen der M.-extensorpollicis-longus-Sehne, eher einer typischen Komplikation der dorsalen Plattenlage. Die Sehnenrupturen sind am ehesten bedingt durch zu weit nach dorsal herausragende Schraubenspitzen, bei den winkelstabilen Schrauben reicht eine monokortikale Verankerung. Drobetz u. Kutscha-Lissberg [2] haben in ihrer Untersuchung von 50 Patienten 6 Rupturen der M.-flexor-pollicis-longus-Sehne gesehen, bedingt durch auftragende Schrauben- köpfe. Rupturen dieser Sehne wurden in unserem Kollektiv nicht beobachtet.

\section{Fazit für die Praxis}

Die volare winkelstabile Plattenosteosynthese stellt eine effiziente Behandlungsoption der distalen Radiusfraktur dar, die insgesamt zu einer hohen Patientenzufriedenheit mit geringer Komplikationsrate führt. Es gilt dabei, die implantatspezifischen Eigenschaften zu beachten.

\section{Korrespondierender Autor Dr. F. Kutscha-Lissberg}

Chirurgische Klinik und Poliklinik, BG-Kliniken Bergmannsheil, Universitätsklinik Bochum, Bürkle-de-la-Camp-Platz 1,44789 Bochum, E-Mail:

Friedrich.Kutscha-Lissberg@ruhr-uni-bochum.de

Interessenkonflikt: Keine Angaben

\section{Literatur}

1. Constantine KJ, Clawson MC, Stern PJ (2002) Volar neutralization plate fixation of dorsally displaced distal radius fractures. Orthopedics 25: 125-128

2. Drobetz H, Kutscha-Lissberg E (2003) Osteosynthesis of distal radius fractures with volar locking screw plate system. Int Orthop 27: 1-6

3. Kamano M, Honda Y, Kazuki K et al. (2002) Palmar plating for dorsally displaced fractures of the distal radius. Clin Orthop 397: 403-408

4. Majetschak M, Koch H, Neudeck F et al. (1997) Unfallursachen und Verletzungsmuster bei In-line-Skating. Unfallchirurgie 23: 171-178

5. Melik N, Togninalli D, Biegger P (1994) Retrospektive Analyse konsekutiv behandelter distaler Radiusfrakturen mit Fixateur externe. Unfallchirurg 97: 645-648

6. Orbay JL, Fernandez DL (2002) Volar fixation for dorsally displaced fractures of the distal radius: a preliminary report.J Hand Surg [Am] 27: 205-215

7. Rueger JM, Linhardt W, Sommerfeldt DW (1998) Differentialindikation zur Behandlung der distalen Radiusfraktur.Trauma Berufskrankh 1: 6-14

8. Schnur DP, Chang B (2000) Extensor tendon rupture after internal fixation of a distal radius fracture using a dorsally placed A07ASIF titanium pi plate. Arbeitsgemeinschaft für Osteosynthesefragen/Association for the Study of Internal Fixation. Ann Plast Surg 44: 564-566

9. Tscherne H, Jahne J (1990) Aktueller Stand der Therapie der distalen Radiusfraktur.Unfallchirurg 93: 157-164

10. Zettl RP, Ruchholtz S, Taeger $G$ et al. (2001) Postoperative Morbidität der operativ behandelten distalen Radiusextensionsfraktur.Unfallchirurg 104: 710-715 